\title{
EXPLORATIONS OF FINNISH MATHEMATICS STUDENTS' BELIEFS ABOUT THE NATURE OF MATHEMATICS
}

\section{Timo Tossavainen, Antti Viholainen, Mervi A. Asikainen and Pekka E. Hirvonen}

Lulea University of Technology

97187 Lulea, Sweden

e-mail: timo.tossavainen@ltu.se

University of Eastern Finland

P. O. Box 111, 80101, Joensuu, Finland

e-mail: antti.viholainen@uef.fi

mervi.asikainen@uef.fi

pekka.e.hirvonen@uef.fi

\begin{abstract}
According to certain surveys made in Germany, mathematics can be seen either as a stagnant structure (formalism-related orientation), a collection of rules, formulas and methods (scheme-related orientation), an active and creative construction process (process-related orientation), or as a method of describing the real world (applicationrelated orientation). We examine how a twenty-item test of students' orientations for students' beliefs on the nature of mathematics, which was developed in the German context, translates to the Finnish culture remaining validity. Altogether 310 first-year students from three Finnish universities took part in the study. We apply confirmatory factor analysis to reveal that a slightly modified version the translated
\end{abstract}

Received: May 18, 2017; Accepted: August 29, 2017

Keywords and phrases: belief, mathematics student, orientation, professional plan, regression. 
questionnaire is applicable also in the Finnish context. Using data collected with the translated questionnaire, we construct a binary regression models to predict students' choosing between a technical and an ordinary university, and how probability that a student chooses teacher's study programme slightly decreases if the static view grows stronger in his/her view of mathematics. Further, we see that all four orientations exist almost equally strong in the Finnish students' beliefs.

\section{Introduction}

Several studies have shown that students' beliefs have a very multifaceted role in the teaching and learning of mathematics (e.g., [10]). These beliefs vary significantly and they concern both the nature of mathematics (e.g., [3, 11, 12]), students themselves as learners of mathematics (e.g., [14]), and even the meaning of individual mathematical notions (e.g., [17]).

The so-called epistemological beliefs concern the structure, certainty, and the source of knowledge [9]. Grigutsch et al. [6] developed a framework for the categorization of such beliefs in mathematics, see also [5]. They studied German mathematics teachers' beliefs about the nature of mathematics and mathematics teaching and learning, and they found four different orientations for teachers' beliefs [5, p. 764]:

- Formalism-related orientation: Mathematics is "an exact science that has an axiomatic basis and is developed by deduction”.

- Scheme-related orientation: Mathematics is "a collection of terms, rules and formulae”.

- Process-related orientation: Mathematics is "a science which mainly consists of problem-solving processes and discovery of structure and regularities".

- Application-related orientation: Mathematics is "a science which is relevant to society and life”. 
The test instrument used by Grigutsch et al. [6, 7] consisted of 77 statements which were classified according to the orientations. Blömeke et al. [1] introduced a shortened version of this Likert-type instrument consisting of twenty items with the six-point scale. More precisely, for each factor, they chose 4-6 items which had the highest factor loadings in [6, 7]. The shortened instrument has also been used in MT21-the Mathematic Teaching in the 21st century study in which six countries participated [16].

Our purpose is to examine whether the shortened instrument is suitable also for exploring first-year university students' epistemological beliefs about the nature of mathematics in the Finnish context. This is not selfevident; according to Peña [13], the establishment of linguistic equivalence through translation techniques is not necessarily sufficient to guarantee the validity of the questionnaire. We also investigate how four orientations can be used to predict students' choosing between a technical and an ordinary university and that of teacher's study programme as they enter a university.

\section{Theoretical Framework}

Students' and teachers' conceptions of mathematics have been studied some times in the past decades. For example, Mura [11, 12] explored over a hundred Canadian mathematicians' and mathematics educators' views of mathematics using a postal survey, and she found thirteen different categories for these conceptions. Crawford et al. [3] studied about 300 firstyear university mathematics students' conceptions of mathematics and they classified them into five different categories, of which two represent fragmented conceptions and three more cohesive conceptions. As these examples show, several different ways to categorize students' and teachers' conceptions of mathematics have been used in previous research. On the other hand, studying beliefs has also been criticized for unfoundedly assuming that students and teachers could easily articulate their beliefs. The critics have proposed viewing beliefs as sensible systems instead of taking them as independent variables. For further information, see [9]. 
As we already said in Section 1, our study is a sequel to [1, 6, 7, 15]. Our aim is to test the translationality of the instrument used in [15]. Blömeke et al. [1] operationalized the four orientations found by Grigutsch et al. [6, 7] using the following twenty statements listed in Table 1 below.

Table 1. The statements concerning the nature of mathematics in [1]

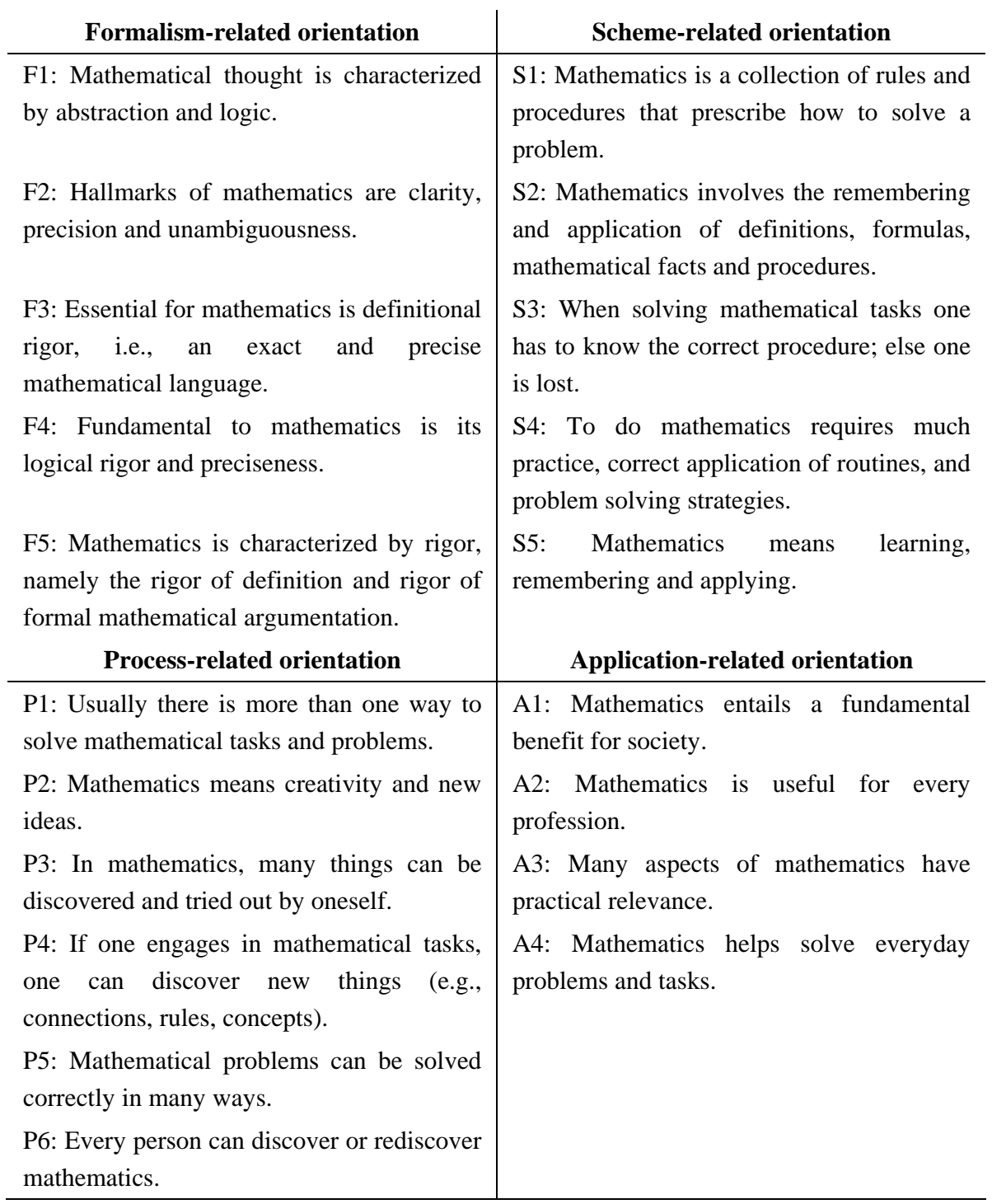


Table 1 shows that details, notations, and rigor are emphasized in the formalism-related orientation. Methods to solve mathematical problems have a key role in the scheme-related orientation, whereas discoveries, inventions, and reasoning are important in the process-related orientation. In the application-related orientation, the benefits of mathematics are emphasized.

Grigutsch et al. [6, 7] found out that there is a positive correlation between the formalism-related and scheme-related orientations, and, analogously, between the process-related and application-related orientations. Hence, they interpreted that the formalism- and scheme-related orientations represent the static aspect of mathematics, whereas the other two orientations refer to the dynamic nature of mathematics. In the static orientations, mathematics is seen as an existing structure that should be found and understood, whereas the dynamic orientations emphasize that mathematics is created by human, not discovered. Such a distinction between static and dynamic beliefs can also be found in [16] and [4], too. Further, in the categorization of [3], both the fragmented and cohesive conceptions may contain application- and scheme-related orientations, but the formalism- and process-orientations are more related to cohesive conceptions. Also, most of Mura's $[11,12]$ thirteen categories can rather easily be organized according to four orientations.

To establish the validity of a translated questionnaire, Peña [13] suggests that a successful translation takes into account, in addition to linguistic equivalence, also functional equivalence, cultural equivalence, and metric equivalence. Functional equivalence refers to avoiding incongruity in meaning in translation, i.e., ensuring that the original and translated questionnaires allow examination of the same construct. Metric equivalence means the equivalence of difficulty in the original and translated in test questions. Cultural equivalence considers the possible ways how respondents from different cultures interpret the underlying meanings of test items.

Hilton and Skrutkowski [8] defined so-called cross-cultural equivalence with content, semantic, technical, criterion, and conceptual dimensions. 
Content equivalence means that the contents of items are relevant in all cultures. Semantic equivalence, resembling functional equivalence in conceptualisation by Peña [13], assumes that the meaning of each item stays the same in a translation process. Technical equivalence refers to the comparability of data collection methods. Interpretation of the data has to be equivalent as well in each culture, this is called criterion equivalence. The last form of equivalence, conceptual equivalence, means that the items measure the same theoretical constructs in each culture. [8, p. 2].

\section{Research Questions}

In [1], both two- and four-dimensional factor models were first extracted using Principal Component Analysis (PCA). In the first case, the factors are interpreted as presenting the static and dynamic aspects of mathematics and, in the latter model, four different orientations for students' beliefs on mathematics. Using Confirmatory Factor Analysis (CFA), they found out that the four-dimensional model fitted their data best. We are also most interested in the four-dimensional models and, consequently, we set our research questions as follows:

(1) How well do the data collected from the Finnish students $(N=310)$ using the translated statements shown in Table 1 support the same fourdimensional model as in [1]?

(2) Is it possible to find any other four-dimensional model to fit the Finnish data?

(3) Is any of the orientations a significant predictor for the students' choosing of university or study programme?

\section{Method}

\section{Instrument}

Our data were collected with a questionnaire that contained a section 
consisting of the Finnish translation of the test by Blömeke et al. [1] with the six-point scales ( 1 = strongly disagree, 6 = strongly agree), see Table 1 , and a section surveying the participants' educational background, i.e., their mathematics grades in upper secondary school, their professional plans etc. The questionnaire was implemented in a web-based form, and all the responses were directly saved to an electronic database.

To establish the validity of translation, linguistic, and functional equivalence were taken into account by involving several translators in the process and repeating the translation of each item until an agreement on the correctness of the end result was achieved. How well cross-cultural equivalence applies to the translation, is a part of our first research question. Metric equivalence should follow from the similar structures of the original and translated questionnaires, given that the individual items are linguistically and functionally equivalent in the original and translated questionnaires.

\section{Sample and the collection of data}

The study was implemented in three Finnish universities where mathematics can be studied as a major subject; 42 students from University A, 172 from University B and 96 from University C answered the questionnaire. All the 310 respondents were just at the beginning of their university studies in mathematics. A and B were multi-disciplinary universities where students majoring in mathematics may qualify either as a subject teacher or mathematician or they can study mathematics as a minor subject; $\mathrm{C}$ was a technical university where all the students have compulsory mathematics courses as part of their first-year studies. All the Finnish-

speaking students who took part in the first course of mathematics in these universities in autumn of 2012 were invited to participate in the study. In A, the students answered the questionnaire during a tutoring lesson, but they worked individually without any additional instruction. Answering the questionnaire was a requirement for passing a compulsory introduction course. Students could reject the use of the responses to the research, but, 
however, no one used this option. In B and C, the students were instructed to fulfil the questionnaire in their own time. Answering the questionnaire was not required, but in $\mathrm{B}$, it was possible to earn one extra point for the first mathematics course by doing so. The total answer rate was almost $100 \%$ in A, $46 \%$ in B, and $15 \%$ in C. In C, there were some technical problems during the data collection, which possibly decreased the answer rate.

For the last research question, the participants of the study were divided into two categories on the basis of their professional orientations as follows:

(1) "Teachers" $(N=72)$. These students were studying in a mathematics teacher education programme or aimed to apply for it. Forty-two of them were ordinary student teachers majoring in mathematics, and twelve will qualify as subject teachers of mathematics and as primary school teachers. Eighteen students were studying mathematics as a minor subject.

(2) “Mathematicians/appliers” $(N=237)$. These students had mathematics as a major or minor subject, and teacher training did not belong to their study programme.

\section{Data analysis}

For studying the model fit, we used confirmatory factor analysis (CFA) with the maximum likelihood method and the Comparative Fit Index (CFI), Root Mean Square Error of Approximation (RSMEA) and Chi-square test. We applied the most commonly used limits for the good model fit: for Chisquare, we expect $p>.05$ (which however occurs only rarely for large data), CFI $>.9$ and RSMEA <.8. Further, we used Oneway ANOVA with post hoc-tests and the standard methods of descriptive statistics.

In addition to using CFA, we computed the sum variables representing four orientations as a means of the corresponding original scales in order the answer the third question. The values of the sum variables range from 1 to 6 similarly as for the individual scales in our questionnaire. The analyses were done using IBM SPSS Statistics 23 and IBM Amos 21 software. 


\section{Results}

We begin by reporting the results of CFA related to the translated twenty-item questionnaire in Table 1 . The model with standardized estimates is shown in Figure 1. The model fit is now only satisfactory

$$
\left(x^{2}(164)=540.57, p<.001 ; \mathrm{CFI}=.78 \text {; RSMEA }=.09\right) \text {. }
$$

The corresponding indices for the German data in [1] are $x^{2}(164)=667.80$, $p<.001$; CFI $=.85$; RSMEA $=.06$ of which CFI is almost a good fit and RSMEA is a good fit. In other words, the model fits the German data somewhat better than the Finnish data. There are also other differences; in our data, the covariance between Formalism and Process is higher than between Formalism and Scheme; in their study, the case is opposite. On the other hand, clearly the highest covariance is between Process and Application in both data.

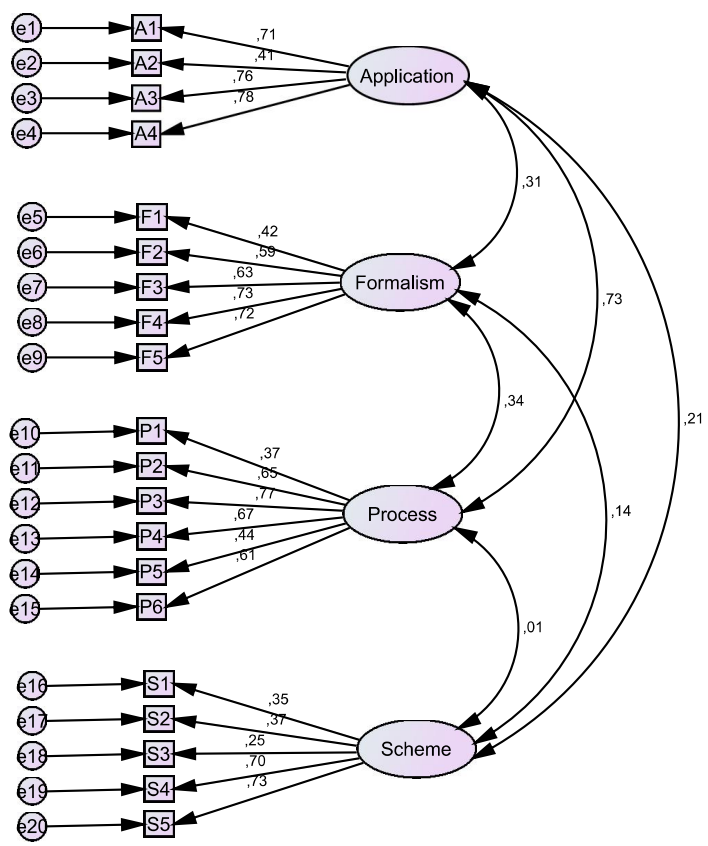

Figure 1. The four-dimensional model of [1] with Finnish data $(N=310)$. 
The most obvious reason for the weaker model fit with the Finnish data is related to the item S3. A deeper analysis revealed that it is loaded almost equally high on Process and Scheme. Other items with relative low loadings are $\mathrm{P} 1$ and $\mathrm{S} 1$.

Figure 2 shows the most applicable model we found using CFA. The fit indices for it are: $x^{2}(113)=245.08, p<.001$; CFI $=.90$; RSMEA $=.06$ meaning a good fit in the light of both CFI and RSMEA. The model was ultimately found by removing the items which had the lowest loadings in Figure 1 ( $\mathrm{P} 1, \mathrm{~S} 1$, and S3). Before that, we also tried to vary from which latent variables these items are loaded, but this did not improve the model fit significantly. Moreover, since we build on the assumption of the validity of the linguistic equivalence between the original and translated items, there are no theoretical reasons for the modifications of this kind as the original model fits the German data rather well.

An interesting observation here is that Application has a moderate covariance with Formalism and Scheme, in addition to having a large covariance with Process. In the German data, these covariances were insignificantly low. On the other hand, in our model, the covariance between Formalism and Scheme is surprisingly low given that they both represent the static view. 


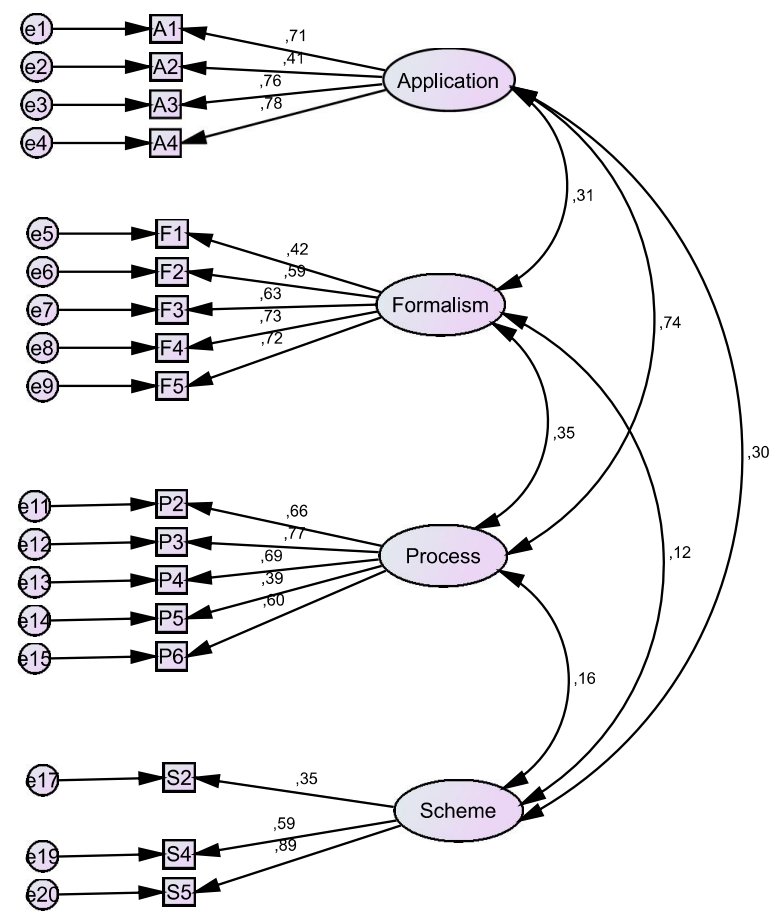

Figure 2. The modified model with Finnish data $(N=310)$.

Next, we examine the descriptive measures for the sum variables representing four orientations (Table 2) and the Pearson correlations between them (Table 3). The sum variables are the means of the observed variables as in Figure 2. They show that all orientations are equally strongly present in the participants' beliefs on the nature of mathematics. Due to the small effect size (Cohen's d), the differences between the means are, in practice, insignificant, yet some of them are statistically significant in Bonferroni's post hoc-test of Oneway ANOVA. The Pearson correlations correspond rather well with those in [1], the most significant difference is that the coefficient between Formalism and Process is a little higher than that between Formalism and Scheme. However, both correlations are weak. 
Table 2. The descriptive measures of the sum variables $(N=301)$

\begin{tabular}{c|c|c|c|c} 
& Application & Formalism & Process & Scheme \\
\hline Mean & 4.79 & 4.81 & 4.54 & 4.73 \\
\hline Std. dev. & .86 & .75 & .84 & .77 \\
\hline Min-Max & $2.00-6.00$ & $2.60-6.00$ & $1.80-6.00$ & $2.00-6.00$ \\
\hline
\end{tabular}

Table 3. The Pearson correlations between the sum variables $(N=301)$

\begin{tabular}{c|c|c|c|c} 
& Application & Formalism & Process & Scheme \\
\hline Application & 1 & $.18 * *$ & $.58 * * *$ & $.20 * *$ \\
\hline Formalism & & 1 & $.25 * * *$ & $.14 *$ \\
\hline Process & & & 1 & .09 \\
\hline Scheme & & & & 1 \\
\hline
\end{tabular}

$*=p<.05, * *=p<.01, * * *=p<.001$

To find out the role of orientations in choosing between a technical and an ordinary university, and between a mathematics teacher's study programme and other mathematical study programme, we modified the model in Figure 2 by first adding the dichotomous variable describing the selection of the university (with $0=$ an ordinary university and $1=a$ technical university), and then, in order to construct another model, a variable describing whether a student has chosen the teacher's study programme (1) or not (0). Further, we added the regression paths from the four latent variables to the dichotomous variable in both models. The results of the analyses of these models are little surprising. Process is the only significant predictor for the selection of the university, and none of the orientations significantly predicts the selection of teacher's study programme.

Next, we continued our analyses by performing a binary logistic analysis where the choosing of the university is the dependent variable and the orientations (sum variables) are the covariates. The results are given in Table 4. They show in more detail, how the increase in the degree of Process 
Mathematics Students’ Beliefs About the Nature of Mathematics 117 implies a decrease in the probability that a student chooses a technical university (since $B_{\text {process }}<0$ ). In other words, the students who emphasize the Process orientation are more likely to go to an ordinary university. The Nagelkerke $R$-squared is not high, yet the percentage of correctly predicted cases is a little above 70 .

Table 4. The binary logistic model predicting the choosing of studying at a technical university $(N=301)$

\begin{tabular}{c|c|c|c|c} 
& $\mathbf{B}$ & S.E. & Sig & $\operatorname{Exp(B)}$ \\
\hline Application & -.13 & .18 & .47 & .88 \\
\hline Formalism & .16 & .18 & .36 & 1.17 \\
\hline Process & -.46 & .19 & .01 & .63 \\
\hline Scheme & .11 & .17 & .51 & 1.12 \\
\hline Constant & .60 & 1.17 & .61 & 1.82 \\
\hline
\end{tabular}

$x^{2}(4)=12.59, p<.05$; Nagelkerke $R^{2}=.06$.

Finally, a similar binary logistic regression analysis with four orientations as predictors did not produce a new insight into the choosing of teacher's study programme. However, we completed our exploration by computing another two sum variables to represent the dynamic and static views of mathematics simply by adding Dynamic $=$ Application + Process and Static $=$ Formalism + Scheme, and performed a binary logistic analysis with these constructs as predictors. The results are shown in Table 5.

Table 5 shows that the static view of mathematics is a statistically significant predictor. Since Nagelkerke $R$-squared is again low, the improvement from the null model to the fitted model is only modest, yet we can conclude that the students emphasizing the static view of mathematics choose less probably a teacher's study programme than those students who do not. 
Table 5. The binary logistic model predicting the choosing of a teacher's study programme $(N=300)$

\begin{tabular}{c|c|c|c|c} 
& B & S.E. & Sig & $\operatorname{Exp(B)}$ \\
\hline Dynamic & .10 & .10 & .30 & 1.10 \\
\hline Static & -.31 & .13 & .01 & .74 \\
\hline Constant & .78 & 1.27 & .54 & 2.17 \\
\hline
\end{tabular}

$x^{2}(2)=6.27, p<.05$; Nagelkerke $R^{2}=.03$

\section{Discussion}

In this study, we examined how a twenty-item test of students' orientations for students' beliefs on the nature of mathematics by Blömeke et al. [1], developed in the German context, translates to the Finnish culture remaining validity. The fit indices of CFA showed that the original twentyitem questionnaire applies somewhat better in the German data than in the Finnish data. However, we observed that by modifying the model by leaving three items (P1, S1, and S3) out, the model fit indices were even slightly better than those for the German data.

We interpret that the differences in the fit indices between the German and Finnish data might be due to the fact that these items do not measure the intended latent variables equally well in the Finnish context. It is for instance possible that conceptual equivalence is not fulfilled in this questionnaire: The Finnish questionnaire and its German counterpart do not measure the same theoretical constructs. Another possible threat to the cross-cultural equivalence is that semantic equivalence or functional equivalence is not fulfilled. Both aspects were however carefully considered during the translation process.

It is quite natural that Process is a significant predictor in choosing between a technical and an ordinary university (Table 4). However, it is slightly surprising that Application did not play any role here as one might expect. We also observed that none of the orientations is a significant predictor for the choosing of teacher's study programme. Assuming that the 
instrument is valid in differentiating different orientations, this result may be good news for mathematics teacher education because it is only positive that prospective mathematics teachers have a broad and versatile view of mathematics.

On the other hand, the binary logistic analysis with the dynamic and static views of mathematics as predictors showed (Table 5) that the students emphasizing the static view of mathematics choose less probably a teacher's study programme than those students who do not emphasize it. This is an interesting result that should be carefully taken into account in mathematics teacher education. If the dynamic view of mathematics dominates student teachers' mathematical beliefs, it easily results in that they also emphasize the procedural knowledge of mathematics in their profession, cf. [17]. This would be a challenge for the objectives that pupils also learn the importance of precise mathematical reasoning and a deeper understanding about mathematical notions in school.

\section{Acknowledgement}

We thank Terhi Hautala, Linda Havola, Juha Oikkonen and Antti Rasila for helping us in collecting the data.

\section{References}

[1] S. Blömeke, A. Felbrich and C. Müller, Future teachers' beliefs on the nature of mathematics, F. Achtenhagen, F. K. Oser and U. Renold, eds., Teachers' Professional Development, Sense Publisher, Rotterdam, 2009, pp. 25-46.

[2] R. B. Cattell, The Scientific Use of Factor Analysis in Behavioral and Life Sciences, Plenum Press, New York, 1978.

[3] K. Crawford, S. Gordon and J. Nicholas, University mathematics students' conceptions of mathematics, Studies in Higher Education 23(1) (1998), 89-94.

[4] P. Ernest, The Impact of Beliefs on the Teaching of Mathematics, P. Ernest, ed., Mathematics Teaching: The State of the Art, Falmer, New York, 1989, pp. 249-253. 
[5] A. Felbrich, C. Müller and S. Blömeke, Epistemological beliefs concerning the nature of mathematics among teacher educators and teacher education students in mathematics, ZDM Mathematics Education 40 (2008), 763-776.

[6] S. Grigutsch, U. Raatz and G. Törner, Einstellungen gegenüber Mathematik bei Mathematiklehrern, Journal für Mathematikdidaktik 19 (1998), 3-45.

[7] S. Grigutsch and G. Törner, World views of mathematics held by university teachers of mathematics science, Schriftemreihe des Fachbereichs Matematik, Preprint 420, Duisburg: Gerhard Mercator University, 1998. Available at http://www.ub.uni-duisburg.de/ETD-db/theses/available/duett-05272002-102811/ unrestricted/mathe121998.pdf

[8] A. Hilton and M. Skrutkowski, Translating instruments into other languages: development and testing processes, Cancer Nursing 25(1) (2002), 1-7.

[9] B. Hofer and P. R. Pintrich, The development of epistemological theories: beliefs about knowledge and knowing and their relation to learning, Review of Educational Research 67(1) (1997), 88-140.

[10] G. C. Leder, E. Pehkonen and G. Törner, eds., Beliefs: A Hidden Variable in Mathematics Education? Kluwer Academic Publisher, Dortrecht, 2002.

[11] R. Mura, Images of mathematics held by university teachers of mathematical sciences, Educational Studies in Mathematics 25(4) (1993), 375-385.

[12] R. Mura, Images of mathematics held by university teachers of mathematics education, Educational Studies in Mathematics 28(4) (1995), 385-399.

[13] E. D. Peña, Lost in translation: methodological considerations in cross-cultural research, Child Development 78(4) (2007), 1255-1264.

[14] B. Roesken, M. Hannula and E. Pehkonen, Dimensions of students' views of themselves as learners of mathematics, ZDM Mathematics Education 43(4) (2011), 497-506.

[15] W. H. Schmidt, S. Blömeke, M. T. Tatto, F. J. Hsieh, K. Cogan, R. T. Houang and J. Schwille, Teacher Education Matters: A Study of Middle School Mathematics Teacher Preparation in Six Countries, Teacher College Press, New York, 2011.

[16] A. H. Schoenfeld, Explorations of students' mathematical beliefs and behavior, Journal for Research in Mathematics Education 20(4) (1989), 338-355.

[17] T. Tossavainen, I. Attorps and P. Väisänen, On mathematics students' understanding of the equation concept, Far East J. Math. Education 6(2) (2011), 127-147. 